TRANSACTIONS OF THE

AMERICAN MATHEMATICAL SOCIETY

Volume 181, July 1973

\title{
FOURIER ANALYSIS ON LINEAR METRIC SPACES
}

\author{
BY
}

\section{J. KUELBS $\left({ }^{1}\right)$}

\begin{abstract}
Probability measures on a real complete linear metric space $E$ are studied via their Fourier transform on $E^{\prime}$ provided $E$ has the approximation property and possesses a real positive definite continuous function $\Phi(x)$ such that $\|x\|>\epsilon$ implies $\Phi(0)-\Phi(x)>c(\epsilon)$ where $c(\epsilon)>0$. In this setting we obtain conditions on the Fourier transforms of a family of tight Borel probabilities which yield tightness of the family of measures. This then is applied to obtain necessary and sufficient conditions for a complex valued function on $E^{\prime}$ to be the Fourier transform of a tight Borel probability on $E$. An extension of the Levy continuity theorem as given by $L$. Gross for a separable Hilbert space is obtained for such metric spaces. We also prove that various Orlicz-type spaces are in the class of spaces to which our results apply. Finally we apply our results to certain Orlicz-type sequence spaces and obtain conditions sufficient for tightness of a family of probability measures in terms of uniform convergence of the Fourier transforms on large subsets of the dual. We also obtain a more explicit form of Bochner's theorem for these sequence spaces. The class of sequence spaces studied contains the $l_{p}$ spaces $(0<p \leq 2)$ and hence these results apply to separable Hilbert space.
\end{abstract}

1. Introduction and basic terminology. Throughout we assume $E$ is a real complete linear metric space. Then $E$ has an invariant metric and letting $\|x\|=d(0, x)$ we see $\|\cdot\|$ has all the properties of a norm, except possibly homogeneity. We will refer to $\|\cdot\|$ as a quasi-norm.

The algebraic dual of $E$ is denoted by $E^{a}$ and the topological dual by $E^{\prime}$.

The Borel sets of a topological space are the minimal sigma-algebra containing the open sets. Here all measures on a topological space are assumed to be Borel measures unless otherwise specified.

A family of probability measures $\left\{\mu_{\alpha}: \alpha \in A\right\}$ on the Borel sets of a topological space is tight if for every $\epsilon>0$ there is a compact set $K$ such that $\mu_{\alpha}(K)>$ $1-\epsilon$ for each $\alpha \in A$. If $\left\{\mu_{\alpha}: \alpha \in A\right\}$ is a tight family of probability measures, then it is obvious that each measure is tight. The converse is not necessarily true. It is also true that every probability measure on the Borel subsets of a complete separable metric space is tight.

Received by the editors July 23, 1971 and, in revised form, August 3, 1972.

AMS (MOS) subject classifications (1970). Primary 60B10, 60B15, 28A40; Secondary 43A35, 46E 30 .

Key words and phrases. Fourier transform, Bochner's theorem, Levy continuity theorem, cylinder set measure, metric approximation property, basis, Orlicz spaces, tight measures.

(1) Supported in part by a NSF Postdoctoral Fellowship and NSF Grant GP 18759.

Copyright $\odot$ 1973, American Mathematical Society 
Suppose $(X, \mathscr{A})$ and $(Y, \mathfrak{B})$ are two measure spaces and $f$ is a measurable map from $(X, \mathfrak{A})$ to $(Y, \mathcal{B})$. Then for each measure $\mu$ on $(X, \mathfrak{A})$ we obtain the measure $\mu^{f}$ on $(Y, B)$ by defining $\mu^{f}(B)=\mu\left(f^{-1}(B)\right)$.

We use the notation ${ }^{t} T$ to denote the transpose of a linear map $T$.

If $\mu$ is a Borel probability on $E$ we define the Fourier transform of $\mu$ on $E^{\prime}$, the topological dual of $E$, by

$$
\hat{\mu}(y)=\int_{E} e^{i\langle y, x\rangle} \mu(d x) .
$$

In particular, if $\mu$ is a tight Borel probability and $E^{\prime}$ contains enough linear functionals to separate the points of $E$, then by the argument in $[8, \mathrm{p} .407$ and p. 414] it follows that $\hat{\mu}$ determines $\mu$ uniquely on the Borel subsets of $E$.

The linear metric space $E$ has a basis $\left\{b_{n}\right\}$ if for each $x \in E$ there is a unique sequence of real numbers $\left\{\beta_{n}\right\}$ such that

$$
\lim _{k}\left\|x-\sum_{n=1}^{k} \beta_{n} b_{n}\right\|=0 .
$$

It then follows that $E$ is separable and we will write the expansion of $x$ as $\sum_{n=1}^{\infty} \beta_{n}(x) b_{n}$ to emphasize that the coefficients generate coordinate functionals on $E$. It is clear that these coordinate functionals are linear and it is known that they are continuous as well.

If $E$ has a basis $\left\{b_{n}\right\}$ we define for $N=1,2, \ldots$ and $x \in E$

$$
P_{N}(x)=\sum_{k=1}^{N} \beta_{k}(x) b_{k}, \quad Q_{N}(x)=x-P_{N}(x) .
$$

We call the sequences $\left\{P_{N}\right\}$ and $\left\{Q_{N}\right\}$ the canonical projections on $E$. Further, since the quasi-norm $\|x\|_{1}=\sup _{N}\left\|P_{N} x\right\|$ generates the same topology on $E$ (a simple application of the open mapping theorem) we have

$$
\left\|P_{N} x\right\| \leq\|x\| \text { and }\left\|Q_{N} x\right\| \leq 2\|x\| \text {. }
$$

Hence the canonical projections on $E$ are equicontinuous and the sequence $\left\{P_{N}\right\}$ converges uniformly to the identity on compact subsets of $E$.

A generalization of the basis concept is that of the approximation property. We say $E$ has the approximation property (metric approximation property) if there is a net of continuous (equicontinuous) operators $\left\{\pi_{\lambda}: \lambda \in \Lambda\right\}$ of $E$ into $E$ such that the range of each $\pi_{\lambda}$ is finite dimensional and $\left\{\pi_{\lambda}: \lambda \in \Lambda\right\}$ converges to the identity map uniformly on compact subsets of $E$.

If there exists a real continuous positive definite function $\Phi(x)$ on $E$ such that for any $\epsilon>0$ and $\|x\|>\epsilon$ we have $\Phi(0)-\Phi(x)>c(\epsilon)$ where $c(\epsilon)>0$, 
then we say that the quasi-norm $\|\cdot\|$ on $E$ is accessible with respect to $\Phi$. We henceforth assume $\Phi$ is such that $\Phi(0)=1$.

The class of all real linear metric spaces $E$ with the approximation property and having an accessible quasi-norm is not known (to me anyway). There are, however, a number of interesting examples such as $L^{P}[0,1](1 \leq p \leq 2)$, any Hilbert space, and various other Orlicz spaces including the real sequence spaces $l_{p}(0<p \leq 2)$. We will discuss these examples in more detail in $\$ \$ 4$ and 6 .

The author is pleased to acknowledge a number of conversations with L. LeCam regarding the results of this paper. In particular, in [7, p. 247] Theorem 2.1 is briefly indicated; my contribution has been to supply some of the details. Finally, the extension of the Fourier transform of a probability measure on $E$ to a random variable on $E^{a}$ is found in [7] and is used in the formulation of the continuity theorems of $\$ 5$. Special cases of this type of extension were previously used in [2] and [4] and the connection is made explicit in $\$ 5$.

2. Tight families of measures on $E$. The result we establish here is essentially that stated (without proof) in [7, p. 247].

Theorem 2.1. Let $E$ be a real complete linear metric space baving the approximation property via the net of operators $\left\{\pi_{\lambda}: \lambda \in \Lambda\right\}$, and assume the quasi-norm on $E$ is accessible with respect to $\Phi$. Let $\left\{\mu_{\alpha}: \alpha \in A\right\}$ be a family of Borel probability measures on $E$ each of which is tight and suppose

(i) $\left\{\mu_{a}{ }^{\pi} \lambda: a \in A\right\}$ is tight for eacb $\lambda \in \Lambda$, and

(ii) $\lim _{s} \sup _{\alpha} \lim _{\lambda} \int_{E^{\prime}}\left[1-\hat{\mu}_{\alpha}\left({ }^{t} \pi_{\lambda} y-{ }^{t} \pi_{s} y\right)\right] P(d y)=0$, where $P$ is the cylinder set measure on $E^{\prime}$ with Fourier transform $\Phi(x)$ on $E$. Then $\left\{\mu_{\alpha}: \alpha \in A\right\}$ is tight.

Before the proof of Theorem 2.1 we provide a basic lemma.

Lemma 2.2. Let $\left\{\mu_{\alpha}: \alpha \in A\right\}$ be a family of Borel probability measures on a complete metric space $(E, d)$. If for every $\epsilon>0$ there exists a Borel map $\pi$ from $E$ into $E$ with the properties

(1) $\sup _{\alpha} \mu_{\alpha}\{x: d(x, \pi x)>\epsilon\}<\epsilon$ and

(2) $\left\{\mu_{\alpha}^{\pi}: \alpha \in A\right\}$ is tight,

then $\left\{\mu_{\alpha}: \alpha \in A\right\}$ is tight in $E$.

Proof. Fix $\epsilon>0$ and choose $\pi$ as in (1) and (2). Then by (2) there exists $x_{1}, \ldots, x_{N}$ in $\pi(E)$ such that $\mu_{\alpha}^{\pi}\left(\bigcup_{j=1}^{N} s\left(x_{j}, \epsilon\right)\right)>1-\epsilon$ for all $\alpha \in A$. Here $s(x, \delta)=\{y: d(y, x)<\delta\}$. Using (1) we obtain $\inf _{\alpha} \mu_{\alpha}\{y: d(y, \pi y)<\epsilon\} \geq 1-\epsilon$, and by (2) a long with our choice of $x_{1}, \ldots, x_{N}$ we have $\inf _{\alpha} \mu_{\alpha}\left(\bigcup_{j=1}^{N}\left\{y: d\left(\pi y, x_{j}\right)<\epsilon\right\}\right)$ $\geq 1-\epsilon$. Hence since $s\left(x_{j}, 2 \epsilon\right) \supseteq\{y: d(y, \pi y)<\epsilon\} \cap\left\{y: d\left(\pi y, x_{j}\right)<\epsilon\right\}$ we obtain 


$$
\inf _{a} \mu_{a}\left(\bigcup_{j=1}^{N} s\left(x_{j}, 2 \epsilon\right)\right)>1-2 \epsilon .
$$

Since $\epsilon>0$ was arbitrary and $E$ is a complete metric space it follows in a standard manner that there exists a compact set $K$ such that $\inf _{a} \mu_{\alpha}(K)>1-\epsilon$. Hence $\left\{\mu_{\alpha}: \alpha \in A\right\}$ is tight as was to be shown.

Proof of Theorem 2.1. In view of (i) and Lemma 2.2 we need only show (1) of Lemma 2.2 holds. Now $\Phi(0)=1$ and since $\|x\|>\epsilon$ implies $1-\Phi(x)>c(\epsilon)$ where $c(\epsilon)>0$ we have

$$
\mu_{\alpha}\left\{x:\left\|x-\pi_{s} x\right\|>\epsilon\right\} \leq \mu_{\alpha}\left\{x: 1-\Phi\left(x-\pi_{s} x\right)>c(\epsilon)\right\}
$$

$$
\leq \frac{1}{c(\epsilon)} \int_{E}\left[1-\Phi\left(x-\pi_{s} x\right)\right] \mu_{\alpha}(d x)=\lim _{\lambda} \frac{1}{c(\epsilon)} \int_{E}\left[1-\Phi\left(\pi_{\lambda} x-\pi_{s} x\right)\right] \mu_{\alpha}(d x)
$$

where the last equality follows since $\mu_{\alpha}$ is tight, $\Phi$ is continuous, and $\| \pi_{\lambda}(x)-$ $x \| \rightarrow 0$ uniformly on compact subsets of $E$. Further, since $\pi_{\lambda}$ is a continuous linear map from $E$ into $E$ with finite dimensional range it follows that the transpose ${ }^{t} \pi_{\lambda}(\lambda \in \Lambda)$ maps $E^{\prime}$ into $E^{\prime}$ and has finite dimensional range. Thus we have

$$
\begin{gathered}
\int_{E}\left[1-\Phi\left(\pi_{\lambda} x-\pi_{s} x\right)\right] \mu_{a}(d x)=\int_{E}\left[\int_{E^{\prime}}\left[1-e^{i\left\langle y, \pi_{\lambda} x-\pi_{s} x\right\rangle}\right] P(d y)\right] \mu_{a}(d x) \\
=\int_{E}\left[\int_{E^{\prime}}\left[1-e^{i\left\langle x,{ }^{t} \pi_{\lambda} y-{ }^{t} \pi_{s} y\right\rangle}\right] P(d y)\right] \mu_{a}(d x) \\
=\int_{E^{\prime}}\left[1-\hat{\mu}_{a}\left({ }^{t} \pi_{\lambda} y-{ }^{t} \pi_{s} y\right)\right] P(d y)
\end{gathered}
$$

where the interchange of integrals and the last integral make sense because the functions are tame functions. Combining (2.1), (2.2), and (ii) we have (1) of Lemma 2.2 and hence $\left\{\mu_{\alpha}: \alpha \in A\right\}$ is tight as was to be shown.

3. Bochner's theorem. The first result is a version of Bochner's theorem for spaces satisfying the conditions of Theorem 2.1 .

Theorem 3.1. Let $E$ be a real complete metric space having the approximation property via the net of operators $\left\{\pi_{\lambda}: \lambda \in \Lambda\right\}$, and assume the quasi-norm on $E$ is accessible with respect to $\Phi$. Let $P$ denote the cylinder set measure on $E^{\prime}$ determined by $\Phi$. T.hen a complex valued function $\Psi$ defined on $E^{\prime}$ is the Fourier transform of a tight Borel probability measure on $E$ iff

(1) $\Psi$ is positive definite on $E^{\prime}$ with $\Psi(0)=1$,

(2) $\Psi$ is continuous on $E^{\prime}$ with respect to the topology generated by uniform 
convergence on compact subsets of $E$, and

(3) $\lim _{s} \sup _{a} \int_{E^{\prime}}\left[1-\Psi\left({ }^{t} \pi_{a} y-{ }^{t} \pi_{a}{ }^{t} \pi_{s} y\right)\right] P(d y)=0$.

Proof. Let $\mu$ be the cylinder set measure on $E$ determined on the algebra of cylinder sets by $\Psi$. Then by (1), $\mu$ exists since (2) implies $\Psi$ is continuous on the finite dimensional subspaces of $E^{\prime}$. For each $\alpha \in \Lambda$ let $\mu_{\alpha}=\mu^{\pi} \alpha$. Then $\mu_{\alpha}$ is a Borel probability measure on $\pi_{\alpha} E \subseteq E$ and has Fourier transform $\hat{\mu}_{\alpha}=\Psi \circ{ }^{t} \pi_{\alpha}$ on $E^{\prime}$. Then for any $s \in \Lambda$ the family $\left\{\mu_{\alpha}^{\pi_{s}}: \alpha \in \Lambda\right\}$ is tight since the measures have support in the finite dimensional subspace $\pi_{s}(E)$ and their Fourier transforms $\hat{\mu}_{\alpha}^{\pi_{s}}=\Psi \circ{ }^{t} \pi_{\alpha}{ }^{\circ} \pi_{s}$ are equicontinuous on ${ }^{t} \pi_{s} E^{\prime}$. To see this last remark simply apply (2) obtaining $\lim _{\alpha} \hat{\mu}_{\alpha}^{\pi_{s}}=\Psi \circ{ }^{t} \pi_{s}$. We now use (3) obtaining for $\alpha$, $s \in \Lambda$ that

$$
\begin{aligned}
\lim _{\boldsymbol{\lambda}} \int_{E^{\prime}} & {\left[1-\hat{\mu}_{\alpha}\left({ }^{t} \pi_{\lambda} y-{ }^{t} \pi_{s} y\right)\right] P(d y) } \\
& =\lim _{\boldsymbol{\lambda}} \int_{E^{\prime}}\left[\int_{E}\left[1-e^{i\left(x,{ }^{t} \pi_{\lambda} y-{ }^{t} \pi_{s} y\right)}\right] \mu_{\alpha}(d x)\right] P(d y) \\
& =\lim _{\boldsymbol{\lambda}} \int_{E}\left[1-\Phi\left(\pi_{\lambda} x-\pi_{s} x\right)\right] \mu_{\alpha}(d x)=\int_{E}\left[1-\Phi\left(x-\pi_{s} x\right)\right] \mu_{\alpha}(d x) \\
& =\int_{E}\left[1-\Phi\left(\pi_{a} x-\pi_{s} \pi_{\alpha} x\right)\right] \mu(d x) \\
& =\int_{E}\left[\int_{E^{\prime}}\left[1-e^{i\left(x, t \pi_{\alpha} y-{ }^{t} \pi_{\alpha}{ }^{t} \pi_{s} y\right\rangle}\right] P(d y)\right] \mu(d x) \\
& =\int_{E^{\prime}}\left[1-\Psi\left({ }^{t} \pi_{a} y-{ }^{t} \pi_{\alpha}{ }^{t} \pi_{s} y\right)\right] P(d y) .
\end{aligned}
$$

The interchange of integrals in (3.1) is allowable since the functions are tame functions whenever the cylinder set measures $\mu$ and $P$ are involved. Thus (3) implies (ii) of Theorem 2.1 and hence by the above $\left\{\mu_{\alpha}: \alpha \in \Lambda\right\}$ is a tight family of Borel probabilities on $E$.

Now ${ }^{t} \pi_{a} y$ converges uniformly to $y$ on compacts of $E$ for each $y \in E^{\prime}$, and hence $\lim _{\alpha} \hat{\mu}_{\alpha}(y)=\Psi(y)$. Thus $\left\{\mu_{\alpha}\right\}$ has as a limit point a tight Borel probability measure $\gamma$ with Fourier transform $\Psi$. To see $\gamma$ is unique simply observe that $\gamma$ is uniquely defined by $\Psi$ on the minimal sigma-algebra $\mathscr{A}$ containing the cylinder sets of $E$. Then since the linear operators $\left\{\pi_{\lambda}: \lambda \in \Lambda\right\}$ are continuous with finite dimensional ranges and they separate points it follows that $E^{\prime}$ separates points in E. Hence we can argue as in [9] to obtain the uniqueness of $\gamma$ on the Borel sets.

Conversely, if $\mu$ is a tight Borel probability on $E$ with Fourier transform $\Psi$ on $E^{\prime}$ then it follows easily that (1) and (2) hold for $\Psi$. Now 


$$
\begin{aligned}
\int_{E^{\prime}}\left[1-\Psi\left({ }^{t} \pi_{a} y-{ }^{t} \pi_{a}{ }^{t} \pi_{s} y\right)\right] P(d y) & =\int_{E}\left[1-\Phi\left(\pi_{\alpha} x-\pi_{s} \pi_{\alpha} x\right)\right] \mu(d x) \\
& =\int_{K}\left[1-\Phi\left(x-\pi_{s} x\right)\right] \mu_{\alpha}(d x)+\delta(K, \alpha, s)
\end{aligned}
$$

where $|\delta(K, \alpha, s)| \leq 2 \mu_{\alpha}(E-K)$. Fix $\epsilon>0$ and choose a compact set such that $\mu(E-K)<\epsilon$. Then $\mu_{\alpha}(K)=\mu^{\pi a}(K)=\mu\left(\pi_{\alpha}^{-1}\left(\pi_{\alpha}(K)\right)\right) \geq \mu(K)>1-\epsilon$ so $|\delta(K, \alpha, s)|$ $\leq 2 \epsilon$. Since $\Phi$ is continuous and $\left\{\pi_{s}\right\}$ converges uniformly to the identity on $K$ it follows that

$$
\lim _{s} \sup _{\alpha} \int_{K}\left[1-\Phi\left(x-\pi_{s} x\right)\right] \mu_{\alpha}(d x)=0
$$

Combining this with (3.2) we have (3) holding.

Corollary 3.2. Let $E$ be a real complete linear metric space with a basis and assume $E$ bas an accessible quasi-norm with respect to $\Phi$. Let $P$ denote the cylinder set measure on $E^{\prime}$ determined by $\Phi$ and $\left\{P_{N}\right\},\left\{Q_{N}\right\}$ the canonical projections on $E$. Then a complex valued function $\Psi$ defined on $E^{\prime}$ is the Fourier transform of a tight Borel probability measure on $E$ iff

(i) $\Psi$ is positive definite on $E^{\prime}$ with $\Psi(0)=1$;

(ii) $\Psi$ is continuous on $E^{\prime}$ with respect to the topology generated by uniform convergence on compact subsets of $E$; and

(iii) $\lim _{N} \sup _{M \geq N} \int_{E^{\prime}}\left[1-\Psi\left({ }^{t} P_{M}{ }^{t} Q_{N} y\right)\right] P(d y)=0$.

The proof of Corollary 3.2 follows immediately from Theorem 3.1 since ${ }^{t} P_{M}$ $-{ }^{t} P_{M}{ }^{t} P_{N}=0$ for $M<N$ and ${ }^{t} P_{M}{ }^{t} Q_{N}$ for $M \geq N$.

4. Applications to various Orlicz spaces. If $\alpha$ is a nonnegative function on $[0, \infty)$ we say $a$ satisfies the $\Delta_{2}$-condition if there exists a $K>0$ such that $\alpha(2 t) \leq K \alpha(t)$ for all $t \geq 0$.

If $(\Omega, \mu)$ is a measure space and $\alpha$ is a nonnegative function defined on $[0, \infty)$ we let $L^{a}(\mu)$ denote the space of all real $\mu$-measurable functions on $\Omega$ such that

$$
\rho(f)=\int_{\mathbf{\Omega}} \alpha(|f(w)|) \mu(d w)<\infty .
$$

If $\alpha$ is increasing on $[0, \infty)$ and satisfies the $\Delta_{2}$-condition then for all $s, t$ we have $\alpha(|s+t|) \leq K[\alpha(|s|)+\alpha(|t|)]$ where $K$ is the constant in the $\Delta_{2}$ condition. Hence, in this situation, the space $L^{a}(\mu)$ is a real linear space.

Now we assume $\alpha$ satisfies the $\Delta_{2}$-condition on $[0, \infty), \alpha$ is continuous and increasing, $\alpha(t)=0$ iff $t=0$, and $\lim _{t \rightarrow \infty} \alpha(t)=+\infty$. Then it is possible to make $L^{\alpha}(\mu)$ into a real complete linear metric space with the quasi-norm

$$
\|f\|_{1}=\inf \{\epsilon>0: \rho(f / \epsilon) \leq \epsilon\},
$$


and we also have that $\rho\left(f_{n}\right) \rightarrow 0$ iff $\left\|f_{n}\right\|_{1} \rightarrow 0$. If, in addition, $\alpha$ is convex on $[0, \infty)$ then $L^{\alpha}(\mu)$ can be made into a Banach space with norm

$$
\|f\|_{2}=\inf \{\epsilon>0: \rho(f / \epsilon) \leq 1\}
$$

and again $\left\|f_{n}\right\|_{2} \rightarrow 0$ iff $\rho\left(f_{n}\right) \rightarrow 0$. Furthermore, let $\beta(t)$ be defined on $[0, \infty)$ and complementary to $\alpha$ in the sense of Young [10, p. 77]. Let $L^{\beta}(\mu)$ denote those $\mu$-measurable functions such that

$$
\rho_{\beta}(g)=\int_{\Omega} \beta(|g(w)|) \mu(d w)<\infty,
$$

and for $f \in L^{a}(\mu)$ define

$$
\|f\|_{3}=\sup _{\rho_{\beta}(g) \leq 1}\left|\int_{\Omega} f g d \mu\right| .
$$

Then $L^{a}(\mu)$ is a Banach space in the norm $\|\cdot\|_{3}$ and $\|\cdot\|_{3}$ is equivalent to the norm $\|\cdot\|_{2}$. We can also prove the Hölder type inequality

$$
\int_{\mathbf{\Omega}}|f g| d \mu \leq\|f\|_{3} N(g) \quad\left(f \in L^{a}(\mu)\right)
$$

where $N(g)=\sup \left\{\int_{\Omega} h g d \mu: \rho(b) \leq 1\right\}$. In particular, if $A$ is a set of finite measure, then

$$
\begin{aligned}
N\left(\chi_{A}\right) & =\sup \left\{\int_{A} b d \mu: \rho(b) \leq 1\right\} \\
& \leq \sup \left\{\mu(A)+\int_{A \cap\{|b| \geq 1\}} b d \mu: \rho(b) \leq 1\right\} \\
& \leq \mu(A)+\sup \left\{\frac{1}{\gamma} \int_{\Omega} a(|b|) d \mu: \rho(b) \leq 1\right\} \leq \mu(A)+\frac{1}{\gamma}
\end{aligned}
$$

where $\gamma>0$ is such that $\alpha(t) \geq \gamma t$ for $t \geq 1$ (such a $\gamma>0$ exists because $\alpha(t)$ is a convex function on $[0, \infty)$ such that $\alpha(t)=0$ iff $t=0)$. For indications as to the proofs of these remarks the reader might consult [3] , [8], and [10]..

We now indicate how the theorems of $\$ \$ 2$ and 3 apply to the spaces $L^{\alpha}(\mu)$.

By the previous remarks $L^{\alpha}(\mu)$ is a real complete linear metric space in the quasi-norm (4.2) or, in case $\alpha$ is convex, in the equivalent norms given by (4.3) and (4.5). Hence to apply our earlier results we must show that $L^{\alpha}(\mu)$ has the approximation property and that the quasi-norm or norms are accessible with respect to some positive definite function in the sense of $\$ 3$.

We first examine the question of accessibility.

Theorem 4.1. Let $\alpha$ be an increasing function on $[0, \infty)$ such that $\lim _{t \rightarrow \infty} \alpha(t)=\infty, \alpha(t)=0$ iff $t=0$, and 


$$
\alpha(t)=\int_{0}^{\infty} x^{-2} \sin ^{2} t x M(d x) \quad(0 \leq t<\infty)
$$

where $M$ is a measure on $[0, \infty)$ which is finite on compact subsets and such that $\int_{1}^{\infty} x^{-2} M(d x)<\infty$. If for each $f \in L^{\alpha}(\mu)$

$$
\Phi(f)=\int_{0}^{\infty} e^{-s \rho(f)} m(d s)
$$

where $m$ is a probability measure on $[0, \infty)$ not concentrated at zero, then $\Phi(f)$ is a real continuous positive definite function on $L^{a}(\mu)$ such that $\Phi(0)=1$. Furthermore, $L^{\alpha}(\mu)$ is a real complete linear metric space in the quasi-norm (4.2) or, in case a is convex, a Banach space in the equivalent norms given by (4.3) and (4.5), and the quasi-norm or norms are accessible with respect to $\Phi$.

Remark 4.1. It is well known that the condition (4.8) along with the conditions on the measure $M$ are equivalent to saying $\exp \{-\alpha(t)\}$ is an infinitely divisable one-dimensional characteristic function.

Proof. First of all observe by (4.8) that $\alpha$ is continuous and $\alpha$ satisfies the $\Delta_{2}$-condition on $[0, \infty)$. Then, by the initial remarks of this section, $L^{\alpha}(\mu)$ is a complete linear metric space (or. Banach space if $\alpha$ is convex) and convergence in $L^{a}(\mu)$ is equivalent to $\rho$-convergence. Hence $\Phi$ is continuous and the quasinorm on norms are accessible by $\Phi$ if we can show $\Phi$ is positive definite on $L^{a}(\mu)$. This follows immediately as in, for example, Theorem 3.2 of [4] since $a$ satisfies (4.8) which is equivalent to $\exp \{-\alpha(t)\}$ being infinitely divisible.

We now examine the spaces $L^{a}(\mu)$ with respect to the approximation property. Our results in this direction are in the next three theorems. Their proofs follow easily from the results of [3] and [11] so are not included.

Theorem 4.2. Let a be a real convex function on $[0, \infty)$ which satisfies the $\Delta_{2}$-condition and $\alpha(t)=0$ iff $t=0$. Then $L^{\alpha}(\mu)$ is a Banach space with the approximation property.

We say a measure space $(\Omega, \mu)$ is separable if there exists a countable collection of sets $\mathcal{C}$ of finite $\mu$-measure having the property that if $A$ is an arbitrary $\mu$-measurable set with $\mu(A)<\infty$ then for each $\epsilon>0$ there exists $C \in \mathcal{C}$ such that $\mu(A-C)+\mu(C-A)<\epsilon$.

Our next theorem provides examples of spaces to which Corollary 3.2 applies.

Theorem 4.3. Let $(\Omega, \mu)$ be a separable $\sigma$-finite measure space and assume $\alpha$ is a real convex function on $[0, \infty)$ which satisfies the $\Delta_{2}$-condition and $\alpha(t)=$ 0 iff $t=0$. Then $L^{a}(\mu)$ is a Banach space with a basis. 
Theorem 4.4. Let $(\Omega, \mu)$ be a purely atomic measure space such that $\mu(\{w\})=$ 1 for $w \in \Omega$. Let $a$ be an increasing continuous function on $[0, \infty)$ satisfying the $\Delta_{2}$-condition, $a(t)>0$ for $t>0$, and sucb that $a(t)$ is asymptotic to $\int_{0}^{\infty} x^{-2} \sin ^{2} t x M(d x)$ at $t=0$ where $M$ is a measure on $[0, \infty)$ which is finite on compact sets and such that $\int_{1}^{\infty} x^{-2} M(d x)<\infty$. Let

$$
\gamma(f)=\int_{\mathbf{\Omega}} \lambda(|f|) d \mu
$$

where

$$
\lambda(t)=\int_{0}^{\infty} x^{-2} \sin ^{2} t x M(d x) \quad(0 \leq t<\infty),
$$

and assume $\lambda(t)$ bounded away from zero on any interval $[\delta, \infty)$ for $\delta>0$. Then $L^{\alpha}(\mu)$ is a real complete linear metric space with the metric approximation property and. $L^{\alpha}(\mu)$ bas an accessible quasi-norm with respect to

$$
\Phi(f)=\int_{0}^{\infty} e^{-s \gamma(f)} \mu(d s)
$$

where $m$ is any probability measure on $[0, \infty)$ not concentrated at zero.

Remark 4.2. It follows easily that if $(\Omega, \mu)$ is a purely atomic measure space and $\Omega$ is countable then the space $L^{\alpha}(\mu)$ in Theorem 4.4 has a basis. For example, the $l^{p}$ spaces $(0<p \leq 2)$ are of this type.

5. The continuity theorem. The continuity theorems we obtain are analogous to those for Hilbert space and the $l_{p}(0<p<2)$ spaces in [2] and [5]. Following Theorem 5.2 we will indicate briefly how our results apply to those in [2] and [5].

Suppose $E$ is a real complete linear metric space and $\mu$ is a tight Borel probability measure on $E$. Then there exists a net of probability measures $\left\{\mu_{b}: b \in B\right\}$ each with mass at only finitely many points in $E$ such that $\left\{\mu_{b}\right\}$ converges weakly to $\mu$. Then if $\mathcal{F}$ is any uniformly equicontinuous and bounded subset of the uniformly continuous functions on $E$ we have

$$
\underset{b}{\lim \sup _{f \in \mathcal{F}}}\left|\int_{E} f d \mu_{b}-\int_{E} f d \mu\right|=0 .
$$

Hence by Proposition 6 of [7] it is possible to extend the definition of the Fourier transform of $\mu$ to $E^{a}$. That is, if $\mu$ is a tight Borel probability measure on $E$ and $\left\{\mu_{b}\right\}$ is a net of probability measure each concentrated at finitely many points then $\tilde{\mu}_{b}(y)=\int_{E} e^{i\langle y, x\rangle} \mu_{b}(d x)$ is defined for each $y \in E^{a}$. Further, in [7] it is shown that $\left\{\tilde{\mu}_{b}\right\}$ is a Cauchy net in quadratic mean with respect to any probability measure $P$ on $E^{a}$ having a continuous Fourier transform $\Phi$ on $E$, and if $\tilde{\mu}=$ l.i.m. ${ }_{b} \tilde{\mu}_{b}$ then 


$$
\int_{E^{a}} \tilde{\mu}(y) P(d y)=\int_{E} \Phi(x) \mu(d x) .
$$

It is quite clear that the definition of $\tilde{\mu}$ as a random variable is independent of the choice of the discrete sequence $\left\{\mu_{b}\right\}$ converging weakly to $\mu$. Moreover, if $E$ has a basis $\left\{b_{n}\right\}$ with canonical projections $\left\{P_{N}\right\}$ then it is easy to show that it is possible to choose the measures $\left\{\mu_{b}\right\}$ so that they all lie in $\bigcup_{N=1}^{\infty} P_{N}(E)$. Hence in the case of the $l_{p}$ spaces $(0<p \leq 2)$ the extended Fourier transform of a measure on $l_{p}$ which was defined in [5] and extended from $l_{p}^{\prime}$ to $R^{\infty}$ (the space of all real sequences) is a special case of that which we work with now. More will be said about $l_{p}$ spaces later.

Theorem 5.1. Suppose $E$ is a real complete linear metric space such that $E$ has the approximation property with respect to the net of operators $\left\{\pi_{\lambda}: \lambda \in \Lambda\right\}$ and $E$ bas an accessible quasi-norm with respect to $\Phi$. Let $P$ denote the probability on $E^{a}$ determined $k y \Phi$. Then a sequence of tight probability measures $\left\{\mu_{k}\right\}$ on $E$ converges weakly to a tight probability measure $\mu$ iff

(1) $\lim \hat{\mu}_{k}=\hat{\mu}$ on $E^{\prime}$, and

(2) for a cofinal subset $\Lambda_{1}$ of $\Lambda$ we bave the sequence of extended trans. forms $\tilde{\mu}_{k}^{I-\pi} \lambda, \lambda \in \Lambda_{1}$, converging in P-probability to $\tilde{\mu}^{I-\pi} \lambda$.

Proof. In view of (1) and the arguments of Theorem 3.1 if we show $\left\{\mu_{k}\right\}$ is a tight sequence of probability measures then $\left\{\mu_{k}\right\}$ has a unique limit point $\mu$ which is tight. Further, since (1) holds we have $\left\{\mu_{k}^{\pi} \lambda\right\}$ converging weakly to $\mu^{\pi} \lambda$ for each $\lambda \in \Lambda$. Hence by Lemma 2.2 it suffices to show for each $\epsilon>0$ there exists a $\lambda \in \Lambda$ such that $\sup _{k} \mu_{k}\left\{x \in E:\left\|x-\pi_{\lambda} x\right\|>\epsilon\right\}<\epsilon$. Fix $\epsilon>0$. Since $E$ has accessible quasi-norm with respect to $\Phi$ we have a $c(\epsilon)>0$ such that

$$
\begin{aligned}
\mu_{k}\left\{x \in E:\left\|x-\pi_{\lambda} x\right\|>\epsilon\right\} \\
\quad \leq \frac{1}{c(\epsilon)} \int_{E}\left[1-\Phi\left(x-\pi_{\lambda} x\right)\right] \mu_{k}(d x) \leq \frac{1}{c(\epsilon)} \int_{E}[1-\Phi(x)] \mu_{k}^{I-\pi}(d x) \\
\quad=\frac{1}{c(\epsilon)} \int_{E}\left\{\int_{E^{a}}\left[1-e^{i\langle x, y\rangle}\right] P(d y)\right\} \mu_{k}^{I-\pi} \lambda(d x)=\frac{1}{c(\epsilon)} \int_{E^{a}}\left[1-\tilde{\mu}_{k}^{I-\pi} \lambda(y)\right] P(d y)
\end{aligned}
$$

where the interchange of integrals is allowable by (5.1). Thus for $\lambda \in \Lambda_{1}$ implies

$$
\begin{aligned}
\varlimsup_{k} \mu_{k}\left\{x \in E:\left\|x-\pi_{\lambda} x\right\|>\epsilon\right\} & \leq \frac{1}{c(\epsilon)} \int_{E^{a}}\left[1-\tilde{\mu}^{I-\pi_{\lambda}}(y)\right] P(d y) \\
& =\frac{1}{c(\epsilon)} \int_{E}\left[1-\Phi\left(x-\pi_{\lambda} x\right)\right]_{\mu}(d x)
\end{aligned}
$$


where the last equality involves an interchange of integrals similar to that in (5.2) and is justified as in (5.1). Since $\mu$ is a tight measure on $E$ and $\left\{\pi_{\lambda}\right\}$ converges uniformly to the identity on compacts we have a $\lambda_{0}$ such that $\lambda \in \Lambda_{1}$ and $\lambda \geq \lambda_{0}$ implies

$$
\frac{1}{c(\epsilon)} \int_{E}\left[1-\Phi\left(x-\pi_{\lambda} x\right)\right] \mu(d x)<\epsilon .
$$

Combining (5.3) and (5.4) we have a $k_{0}$ such that $k \geq k_{0}$ and $\lambda \geq \lambda_{0}, \lambda \in \Lambda_{1}$ implies

$$
\sup _{k \geq k_{0}} \mu_{k}\left\{x \in E:\left\|x-\pi_{\lambda} x\right\|>\epsilon\right\}<\epsilon .
$$

Since each $\mu_{k}$ is tight it follows that there exists a $\lambda_{1}$ such that $\lambda \geq \lambda_{1}, \lambda \in \Lambda_{1}$ implies $\sup _{k} \mu_{k}\left\{x \in E:\left\|x-\pi_{\lambda} x\right\|>\epsilon\right\}<\epsilon$. Thus $\left\{\mu_{k}\right\}$ is tight and as remarked above $\left\{\mu_{k}\right\}$ converges weakly to some tight probability $\mu$.

Now assume $\left\{\mu_{k}\right\}$ converges weakly to $\mu$. Then (1) holds and to show (2) holds we show $\left.\tilde{\mu}_{k}^{I-{ }^{I}} \lambda\right\}$ converges in mean square to $\tilde{\mu}^{I-\pi} \lambda$ for each $\lambda \in \Lambda$. Fix $\lambda \in \Lambda$ and let $\Psi_{k}=\tilde{\mu}_{k}^{I-\pi} \lambda, \Psi=\tilde{\mu}^{I-\pi} \lambda$. Then

$$
\int_{E^{a}}\left|\Psi_{k}-\Psi\right|^{2} d P=\int_{E^{a}} \Psi_{k} \bar{\Psi}_{k} d P-\int_{E^{a}} \Psi_{k} \bar{\Psi} d P-\int_{E^{a}} \bar{\Psi}_{k} \Psi d P+\int_{E^{a}} \Psi \bar{\Psi} d P
$$

and since each measure $\mu_{k}$ is tight it follows that $\mu_{k}^{I-{ }^{-} \lambda}$ is the weak limit of discrete measures $\left\{\mu_{k, b}: b \in B\right\}$. Hence, as indicated prior to (5.1), $\left\{\tilde{\mu}_{k, b}\right\}$ converges in mean square to $\Psi_{k}$ and the functions being bounded implies

$$
\begin{aligned}
& A=\lim _{k} \int_{E} \Psi_{k} \bar{\Psi}_{k} d P=\lim _{k} \int_{E}\left[\lim _{b} \int_{E} \int_{E} e^{i(y, x-z)} \mu_{k, b}(d x) \mu_{k, b}(d z)\right] d P(y) \\
& =\lim _{k} \lim _{b} \int_{E} \int_{E} \Phi(x-z) \mu_{k, b}(d x) \mu_{k, b}(d z) \\
& =\lim _{k} \int_{E} \int_{E} \Phi(x-z) \mu_{k}^{I-\pi} \lambda(d x) \mu_{k}^{I-\pi} \lambda(d z) \\
& =\int_{E} \int_{E} \Phi(x-z) \mu^{I-\pi} \lambda(d x) \mu^{I-\pi} \lambda(d z)
\end{aligned}
$$

where the last equality follows because $\left\{\mu_{k} \times \mu_{k}\right\}$ converges weakly to $\mu \times \mu$. Similarly, $\lim _{k} \int_{E}{ }^{a} \Psi_{k} \bar{\Psi} d P=\lim _{k} \int_{E} \bar{\Psi}_{k} \Psi d P=\int_{E} a \Psi \bar{\Psi} d P=A$ and hence by (5.6) we have (2) holding.

In Theorem 5.1 it is condition (2) which is somewhat unusual. For example, in [2] and [5], (2) was replaced by the simpler condition that $\tilde{\mu}_{k}$ converges in 
probability to $\tilde{\mu}$. In our next theorem we are able to obtain this result provided we make additional assumptions on $E$ and $\Phi$. We remark, however, that these assumptions are available in the setting of [2] and [5] as we will show after Theorem 5.2.

Theorem 5.2. Suppose $E$ is a real complete linear metric space and that $E$ bas the metric approximation property via the net of operators $\left\{\pi_{\lambda}: \lambda \in \Lambda\right\}, E$ has an accessible quasi-norm with respect to $\Phi$, and for each $x \in E, \gamma>0$, and $\lambda$ in a cofinal subset $\Lambda_{1}$ of $\Lambda, \Phi\left(\gamma \pi_{\lambda} x+\left(x-\pi_{\lambda} x\right)\right) \leq \Phi\left(x-\pi_{\lambda} x\right)$. Let $P$ be the probability measure on $E^{a}$ determined by $\Phi$ and assume $P^{T} \gamma, \lambda$ is absolutely continuous with respect to $P$ where $T_{\gamma, \dot{\lambda}}=\gamma^{t} \pi_{\lambda}+\left(I-{ }^{t} \pi_{\lambda}\right)$ for $\gamma>0$ and $\lambda \in \Lambda_{1}$. Then a sequence of tight probability measures $\left\{\mu_{k}\right\}$ converges weakly to a tight probability measure $\mu$ iff

(1) $\lim _{k} \hat{\mu}_{k}=\hat{\mu}$ on $E^{\prime}$,

(2) $\left\{\tilde{\mu}_{k}\right\}$ converges in P-probability to $\tilde{\mu}$ on $E^{a}$.

Proof. The proof of the necessity of (1) and (2) is exactly as in Theorem 5.1. To show that (1) and (2) are sufficient it suffices (as is explained in the proof of Theorem 5.1) to show that for $\epsilon>0$ there exists a $\lambda$ such that $\sup _{k} \mu_{k}\{x \in E$ : $\left.\left\|x-\pi_{\lambda} x\right\|>\epsilon\right\}<\epsilon$. Now let $\epsilon>0$ be given. Then there exists a $c(\epsilon)>0$ such that for $\lambda \in \Lambda_{1}$ and $y>0$

$$
\begin{aligned}
\mu_{k}\left\{x:\left\|x-\pi_{\lambda} x\right\|>\epsilon\right\} & \leq \mu_{k}\left\{x: 1-\Phi\left(x-\pi_{\lambda} x\right)>c(\epsilon)\right\} \\
& \leq \mu_{k}\left\{x: 1-\Phi\left(\gamma \pi_{\lambda} x+\left(x-\pi_{\lambda} x\right)\right)>c(\epsilon)\right\} \\
& =\frac{1}{c(\epsilon)} \int_{E}\left[1-\Phi\left(\gamma \pi_{\lambda} x+\left(x-\pi_{\lambda} x\right)\right)\right] \mu_{k}(d x) \\
& =\frac{1}{c(\epsilon)} \int_{E}\left\{\int_{E^{a}}\left[1-e^{i\left\langle y, \gamma \pi_{\lambda} x+\left(x-\pi_{\lambda} x\right)\right\rangle}\right] P(d y)\right\} \mu_{k}(d x) \\
& =\frac{1}{c(\epsilon)} \int_{E}\left\{\int_{E^{a}}\left[1-e^{i\langle T} \gamma, \lambda^{y, x\rangle}\right] P(d y)\right\} \mu_{k}(d x) \\
& =\frac{1}{c(\epsilon)} \int_{E}\left\{\int_{E^{a}}\left[1-e^{i\langle y, x\rangle}\right] P^{T} \gamma, \lambda(d y)\right\} \mu_{k}(d x) \\
& =\frac{1}{c(\epsilon)} \int_{E^{a}}\left[1-\tilde{\mu}_{k}(y)\right] P^{T} \gamma, \lambda(d y)
\end{aligned}
$$

where the last equality follows as in (5.1). Thus, by (2) and that $P^{T} \gamma, \lambda<<$, 


$$
\begin{aligned}
\varlimsup_{k} \mu_{k}\left\{x:\left\|x-\pi_{\lambda} x\right\|>\epsilon\right\} & \leq \frac{1}{c(\epsilon)} \int_{E^{a}}[1-\tilde{\mu}(y)] P^{T} \gamma, \lambda(d y) \\
& =\frac{1}{c(\epsilon)} \int_{E}\left[1-\Phi\left(\gamma \pi_{\lambda} x+\left(x-\pi_{\lambda} x\right)\right)\right]_{\mu}(d x) .
\end{aligned}
$$

Since $\mu$ is tight and the operators $\left\{\pi_{\lambda}: \lambda \in \Lambda\right\}$ are equicontinuous it follows from the continuity of $\Phi$ on $E$ that there exists $\lambda_{0}, \gamma_{0}>0$ such that for $\lambda \in \Lambda_{1}, \lambda>\lambda_{\theta}$, and $0<y<\gamma_{0}$

$$
\int_{E}\left[1-\Phi\left(y \pi_{\lambda} x+\left(x-\pi_{\lambda} x\right)\right)\right] \mu(d x)<\epsilon \cdot c(\epsilon)
$$

Thus there exists a $k_{0}$ such that for $\lambda \in \Lambda_{1}$ and $\lambda>\lambda_{0}$

$$
\sup _{k \geq k_{0}} \mu_{k}\left\{x:\left\|x-\pi_{\lambda} x\right\|>\epsilon\right\}<\epsilon .
$$

The proof of the sufficiency is now completed as in Theorem 5.1.

Suppose $E$ has a basis $\left\{b_{n}\right\}$ with coefficient functionals $\left\{\beta_{n}\right\}$. Then the map $x \rightarrow\left\{\beta_{n}(x)\right\}$ is injective from $E$ into $R^{\infty}$, the space of all real sequences, and $E$ can be viewed as a sequence space. Now each sequence $\left\{y_{i}\right\}$ in $R^{\infty}$ induces a linear map $y$ on $E$ by defining

$$
y\left(\sum_{j=1}^{k} a_{j} b_{j}\right)=\sum_{j=1}^{k} a_{j} y_{j}
$$

and then extending $y$ linearly to all of $E$. Hence $R^{\infty}$ can be injected into the algebraic dual $E^{a}$ and the dual $E^{\prime}$ of $E$ can also be viewed as a sequence space via the injective mapping $y \rightarrow\left\{y\left(b_{j}\right)\right\}$ from $E^{\prime}$ to $R^{\infty}$. Now if $\Phi$ is a continuous positive definite function on $E$ there is then a unique Borel probability on $R^{\infty}$ and we can work with $R^{\infty}$ instead of $E^{a}$. For example if $\hat{\mu}$ is the Fourier transform of a probability measure $\mu$ on $E$ and $E^{\prime}$ is viewed as a subset of $R^{\infty}$, then $\hat{\mu}$ can be extended as a random variable to $R^{\infty}$ as in the discussion around (5.1). We denote this extension by $\tilde{\mu}$ as before.

We now fix some notation for the following corollary which implies the continuity theorem of [5] for the $l_{p}$ spaces, $0<p \leq 2$.

Let $E_{\alpha}$ denote the space of real sequences $x=\left\{x_{i}\right\}$ such that $\sum_{i=1}^{\infty} \alpha\left(\left|x_{i}\right|\right)<\infty$ where $\alpha$ is an increasing continuous function on $[0, \infty)$ satisfying the $\Delta_{2}$ condition, $\alpha(t)>0$ for $t>0$, and such that $\alpha(t)$ is asymptotic to $\lambda(t)=$ $\int_{0}^{\infty} x^{-2} \sin ^{2} t x M(d x) \quad(0 \leq t<\infty)$ at $t=0$ where $M$ is a measure on $[0, \infty)$ which is finite on compact sets and such that $\int_{1}^{\infty} x^{-2} M(d x)<\infty$. Let $\Phi(x)=e^{-\gamma(x)}$ where

$$
\gamma(x)=\sum_{i=1}^{\infty} \lambda\left(\left|x_{i}\right|\right) \quad\left(x \in E_{a}\right) .
$$


Further, assume $\lambda(t)$ is bounded away from zero on $[\delta, \infty)$ for each $\delta>0$ and that $e^{-\lambda(|t|)}$ is Lebesgue integrable on $(-\infty, \infty)$. Let $P$ denote the product probability on $R^{\infty}$ determined by $\Phi(x)$ and let $T_{\gamma, N^{\prime}}=\gamma^{t} P_{N}+\left(I-{ }^{t} P_{N}\right)$ be defined on $R^{\infty}$ where $\left\{P_{N}\right\}$ denotes the canonical projections in $E_{\alpha}$. Since $e^{-\lambda(|t|)}$ is integrable it follows that $P^{T} \gamma, N<<$ for each $\gamma>0$ and integer $N$. Let $\mu$ denote a probability measure on $E_{\alpha}$. Then $\mu$ has an extended Fourier transform $\tilde{\mu}$ on $R^{\infty}$. This extension can be obtained as in the discussion around (5.1), or by the method employed in [5] when $\alpha(t)=t^{p}(0<p \leq 2)$. In both cases we obtain the same random variables on $R^{\infty}$.

Corollary 5.1. Let $\alpha, \lambda, \Phi$, and $E_{\alpha}$ be defined as above, and let $P$ denote the probability on $R^{\infty}$ determined by $\Phi$. Then a sequence of probability measures $\left\{\mu_{k}\right\}$ on $E_{a}$ converges weakly to a probability measure $\mu$ on $E_{a}$ iff

(1) $\lim \hat{\mu}_{k}=\hat{\mu}$ on $E^{\prime}$, and

(2) $\left\{\tilde{\mu}_{k}\right\}$ converges in P-prokability to $\tilde{\mu}$ on $R^{\infty}$.

Proof. The corollary follows directly from the argument used in Theorem 5.2 by replacing the algebraic dual of $E_{\alpha}$ by $R^{\infty}$ as we have indicated, and using the canonical projections $\left\{P_{N}\right\}$ in $E_{\alpha}$. That is, $E_{a}$ has the metric approximation property with respect to the canonical projections $\left\{P_{N}\right\}$; for $x \in E_{\alpha}, \gamma>0$ and each $P_{N}, \Phi\left(\gamma P_{N} x+\left(x-P_{N} x\right)\right) \leq \Phi\left(x-P_{N} x\right)$; and if $T_{\gamma, N}$ is as above then $P^{T} y, N$ is absolutely continuous with respect to $P$. Hence the argument used in Theorem 5.2 is valid.

Remark 5.1. If $\alpha(t)=\lambda(t)=t^{P}, 0<t \leq 2$, then Corollary 5.1 immediately implies Theorem 3.1 of [5].

6. Application to sequence spaces. Throughout this section we will be dealing with the sequence space $E_{\alpha}$ consisting of real sequences $\left\{x_{i}\right\}$ such that $\sum_{i=1}^{\infty} \alpha\left(\left|x_{i}\right|\right)<\infty$ where we assume

(6.1) $\alpha$ is an increasing continuous function on $[0, \infty)$ satisfying the $\Delta_{2}$ condition.

(6.2) $\alpha(t)>0$ for $t>0$ and $\alpha(t)$ is asymptotic to $\lambda(t)=\int_{0}^{\infty} x^{-2} \sin ^{2} t x M(d x)(0 \leq$ $t<\infty)$ at $t=0$ where $M$ is a measure on $[0, \infty)$ which is finite on compact sets and such that $\int_{1}^{\infty} x^{-2} M(d x)<\infty$, and $\lambda(t)$ is bounded away from zero on $[\delta, \infty)$ for each $\delta>0$.

(6.3) $\Phi(x)=e^{-\gamma(x)}$ where $\gamma(x)=\sum_{i=1}^{\infty} \lambda\left(\left|x_{i}\right|\right)$ and $x=\left\{x_{i}\right\} \in E_{\alpha}$.

(6.4) $P_{\lambda}$ is the cylinder set probability on $E_{\alpha}^{\prime}$, the space of continuous linear functionals on $E_{\alpha}$, which is determined by the continuous positive definite function $\Phi(x)$ on $E_{\alpha}$.

As pointed out in $\$ 4, E_{\alpha}$ is a complete linear metric space and, by Theorem 4.4, $\Phi(x)$ is a positive definite continuous function on $E_{\alpha}$ such that the quasi- 
norm of $E_{\alpha}$ is accessible with respect to $\Phi$. In particular, if $\alpha(t)=\lambda(t)=t^{p}$, $0<p \leq 2$, we have $E_{\alpha}=l^{p}$ and $P_{\lambda}$ is the canonical stable cylinder set measure on $l^{p^{\prime}}$.

An operator $T$ on $E_{\alpha}^{\prime}$ to $E_{a}$ is said to be an $S_{\alpha}$-operator if $T$ can be represented by an infinite symmetric positive definite matrix $\left(t_{i j}\right)$ such that $\sum_{i=1}^{\infty} a\left(t_{i i}^{1 / 2}\right)<\infty$. The $\tau_{\alpha}^{-t o p o l o g y}$ on $E_{\alpha}^{\prime}$ is that generated by the seminorms $(T x, x)^{1 / 2}$ as $T$ varies over the $S_{\alpha}$-operators. To verify that the $S_{a}$ operators map $E_{\alpha}^{\prime}$ to $E_{\alpha}$ simply note that since $\left(t_{i j}\right)$ is positive definite we have $\left(t_{i j}\right) \leq t_{i i}^{1 / 2} t_{j j}^{1 / 2}$ for $i, j=1,2, \ldots$ and hence for $x=\left\{x_{j}\right\} \in E_{a}^{\prime}$ we have $T x=\left\{\sum_{j=1}^{\infty} t_{i j} x_{j}\right\}$ in $E_{\alpha}$. That is, since a satisfies the $\Delta_{2}$-condition we have

$$
\sum_{i=1}^{\infty} \alpha\left(\left|\sum_{j=1}^{\infty} t_{i j} x_{j}\right|\right) \leq \sum_{i=1}^{\infty} \alpha\left(d t_{i i}^{1 / 2}\right)<M \sum_{i=1}^{\infty} \alpha\left(t_{i i}^{1 / 2}\right)<\infty
$$

where $d=\sum_{j=1}^{\infty} t_{j j}^{1 / 2}\left|x_{j}\right|<\infty$ since $\left\{t_{j j}^{1 / 2}\right\} \in E_{\alpha}$ and $\left\{\left|x_{j}\right|\right\} \in E_{\alpha}^{\prime}$. Hence $T$ maps $E_{a}^{\prime}$ to $E_{a}$ and $(T x, x)^{1 / 2}$ is a seminorm on $E_{a}^{\prime}$.

Lemma 6.1. Let a satisfy (6.1) and (6.2) and $\lambda$ be as in (6.2). Let $Z_{1}$, $Z_{2}, \ldots$ be independent identically distributed random variables defined on the probability space $(\Omega, \mathcal{F}, P)$ with common Fourier transform $e^{-\lambda(|t|)},-\infty<t<\infty$. Let $T=\left(t_{i j}\right)$ be a $S_{a}$-operator and suppose $T_{n, m}(Z)=\sum \sum_{i, j=n+1}^{m} t_{i j} Z_{i} Z_{j}$. Then for each $\epsilon>0$

$$
\lim _{m>n} P\left(T_{n, m}(Z)>\epsilon\right)=0 .
$$

Proof. Let $c_{n}=\left|t_{n n}\right|^{-1 / 2}$ for $n=1,2, \ldots$ and define $Y_{n}=Z_{n}$ if $\left|Z_{n}\right| \leq c_{n}$ and $Y_{n}=0$ otherwise. Then $P\left(Y_{n}=Z_{n}\right)=P\left(\left|Z_{n}\right|>c_{n}\right)$ and by [6, p. 196], since $\alpha \sim \lambda$ and $\alpha$ is increasing on $[0, \infty)$,

$$
\begin{aligned}
& P\left(\left|Z_{n}\right|>c_{n}\right) \leq 7 c_{n} \int_{0}^{1 / c_{n}}\left[1-e^{-\lambda(v)}\right] d v \\
& \sim 7 c_{n} \int_{0}^{1 / c_{n}}\left[1-e^{-\alpha(v)}\right] d v \leq 7 c_{n} \alpha\left(\frac{1}{c_{n}}\right) \int_{0}^{1 / c_{n}} d v=7 a\left(\left|t_{n n}\right|^{1 / 2}\right) .
\end{aligned}
$$

Since $\left\{\left|t_{n n}\right|^{1 / 2}\right\} \in E_{a}$ it follows from the Borel-Cantelli lemma that

$$
P\left\{\lim _{m>n}\left[T_{n, m}(Z)-T_{n, m}(Y)\right]=0\right\}=1
$$

Now for $\epsilon>0$

$$
P\left(T_{n, m}(Z)>\epsilon\right) \leq P\left(T_{n, m}(Y)>\epsilon / 2\right)+P\left(\left|T_{n, m}(Z)-T_{n, m}(Y)\right|>\epsilon / 2\right)
$$


and by (6.6) the last term can be made arbitrarily small by taking $m>n$ sufficiently large. Further,

$$
P\left(T_{n, m}(Y)>\epsilon / 2\right) \leq \frac{2}{\epsilon} \sum_{i, j=n+1}^{m} \sum_{i j} E\left(Y_{i} Y_{j}\right)=\frac{2}{\epsilon} \sum_{i=n+1}^{m} t_{i i} E\left(Y_{i}^{2}\right)
$$

where the last equality follows since the $\left\{Y_{j}\right\}$ are independent and symmetric. Now by $[6, p .196]$

$$
E\left(Y_{n}^{2}\right) \leq 3\left|t_{n n}\right|^{-1}\left[1-e^{-\lambda\left(\left|t_{n n}\right|^{1 / 2}\right)}\right] \leq M \alpha\left(\left|t_{n n}\right|^{1 / 2}\right) /\left|t_{n n}\right|
$$

where $M$ is a nonnegative constant. Combining (6.7), (6.8), and (6.9) we have (6.5).

With the aid of Lemma 6.1 we now provide sufficient conditions for conditional compactness of families of probability measures on $E_{\alpha}$ in terms of the corresponding Fourier transforms on $E_{\alpha}^{\prime}$. Of course the results of $\$ \$ 2$ and 5 are already along these lines and apply to the $E_{a}$ spaces, but the results we obtain now are of a somewhat different type.

Theorem 6.1. Let $\left\{\mu_{k}\right\}$ be a sequence of probability measures on $E_{\alpha}$ and assume (6.1), (6.2), (6.3), and (6.4) bold. For each $S_{\alpha}$-operator $T$ let

$$
U_{T}=\left\{x \in E_{a}^{\prime}:(T x, x) \leq 1\right\} .
$$

If for each $\epsilon>0$ there exists an $S_{a}$-operator $T_{\epsilon}$ and an integer $k_{\epsilon}$ such that

$$
\sup _{k \geq k \epsilon} \sup _{x \in U_{T}}\left|1-\hat{\mu}_{k}(x)\right| \leq \epsilon,
$$

then $\left\{\mu_{k}\right\}$ is conditionally compact.

Proof. Let $\left\{P_{m}\right\},\left\{Q_{m}\right\}$ denote the canonical projections on $E_{a}$. Since the hypotheses imply that $\left\{\hat{\mu}_{k}\right\}$ is equicontinuous at zero in $P_{m} E_{\alpha}^{\prime}$ for each integer $m$ we need only verify (ii) of Theorem 2.1 to show $\left\{\mu_{k}\right\}$ is tight and hence conditionally compact.

Let $\epsilon>0$ be given. Choose an $S_{\alpha}$-operator $T_{\epsilon}$ and $k_{\epsilon}$ such that (6.11) holds. Then

$$
\begin{aligned}
& \lim _{n} \sup _{k \geq k_{\epsilon}} \lim _{m} \int_{E_{a}^{\prime}}\left[1-\hat{\mu}_{k}\left({ }^{t} P_{m} y-{ }^{t} P_{n} y\right)\right] P_{\boldsymbol{\lambda}}(d y) \\
& =\underset{n}{\lim \sup _{k \geq k} \lim _{\epsilon}} \int_{E_{a}^{\prime}}\left[1-\hat{\mu}_{k}\left({ }^{t} P_{m}{ }^{t} Q_{n} y\right)\right] P_{\lambda}(d y) \\
& \leq \epsilon+\lim _{n} \lim _{m} 2 P_{\lambda}\left\{y \in E_{\alpha}^{\prime}:\left(T_{\epsilon}{ }^{t} P_{m}{ }^{t} Q_{n} y,{ }^{t} P_{m}{ }^{t} Q_{n} y\right)>1\right\} .
\end{aligned}
$$


In view of the definition of $P_{\lambda}$ and Lemma 6.1 we have

$$
\underset{n}{\lim } \lim _{m} P_{\lambda}\left\{y \in E_{a}^{\prime}:\left(T_{\epsilon}{ }^{t} P_{m}{ }^{t} P_{n} y,{ }^{t} P_{m}{ }^{t} P_{n} y\right)>1\right\}=0 .
$$

Now each $\mu_{k}$ is tight and $E_{a}$ has the approximation property with respect to $\left\{P_{m}\right\}$ so

$$
\begin{aligned}
& \underset{n}{\lim } \sup _{\substack{k<k_{\epsilon} \\
\lim _{m}}} \int_{E_{a}^{\prime}}\left[1-\hat{\mu}_{k}\left({ }^{t} P_{m} y-{ }^{t} P_{\cdot n} y\right)\right] P_{\lambda}(d y) \\
& \quad=\underset{n}{\lim \sup _{k<k_{\epsilon}} \lim _{m}} \int_{E_{\alpha}}\left[1-\Phi\left(P_{m} x-P_{n} x\right)\right] \mu_{k}(d x)=0 .
\end{aligned}
$$

Combining (6.12), (6.13), and (6.14) we have

$$
\lim _{n} \sup _{k} \lim _{m} \int_{E}^{\prime}\left[1-\hat{\mu}_{k}\left({ }^{t} P_{m} y-{ }^{t} P_{n} y\right)\right] P_{\lambda}(d y) \leq \epsilon
$$

so $\left\{\mu_{k}\right\}$ is tight as was to be proved.

Remark 6.1. Let $\left\{\mu_{a}: a \in A\right\}$ be a family of probability measures on $E_{a}$ and suppose $\left\{\hat{\mu}_{a}: a \in A\right\}$ is equicontinuous at zero in the $\tau_{a}$-topology. Then (6.11) holds for all $a \in A$ and some $S_{\alpha}$-operator $T_{a}$ and hence $\left\{\mu_{a}: a \in A\right\}$ is conditionally compact.

Theorem 6.2. Let $\left\{\mu_{k}\right\}$ be a sequence of probability measures on $E_{a}$ and assume (6.1), (6.2), (6.3), and (6.4) bold. Suppose for each $\epsilon>0$ there exists an integer $k_{\epsilon}$ and an $S_{a}$-operator $T_{\epsilon}$ such that, for $\left(T_{\epsilon} x, x\right) \leq 1$ and $k \geq k_{\epsilon^{\prime}}$

$$
\left|\hat{\mu}_{k}(x)-\hat{\mu}_{k}(x)\right|<\epsilon \text {. }
$$

Then $\left\{\mu_{k}\right\}$ is conditionally compact.

Proof. Again it suffices to verify (ii) of Theorem 2.1. Let $\epsilon>0$ be given and choose an $S_{\alpha}$-operator $T_{\epsilon}$ such that

$$
\sup _{k \geq k} \sup _{\epsilon}\left|\dot{\hat{\mu}}_{\epsilon}(x)-\hat{\mu}_{k_{\epsilon}}(x)\right|<\epsilon .
$$

Then

$$
\begin{aligned}
& \underset{n}{\lim } \sup _{k \geq k} \lim _{m} \int_{E_{\alpha}^{\prime}}\left[1-\hat{\mu}_{k_{\epsilon}}\left({ }^{t} P_{m}{ }^{t} Q_{n} y\right)\right] P_{\lambda}(d y) \\
& \leq \underset{n}{\leq} \lim _{m} \lim _{E_{\alpha}^{\prime}}\left[1-\hat{\mu}_{k_{\epsilon}}\left({ }^{t} P_{m}{ }^{t} Q_{n} y\right)\right] P_{\lambda}(d y) \\
& \quad+\epsilon+2 \underset{n}{2 \lim \lim _{m} P_{\lambda}\left\{y \in E_{\alpha}^{t}:\left(T_{\epsilon}{ }^{t} P_{m}{ }^{t} Q_{n} y,{ }^{t} P_{m}{ }^{t} Q_{n} y\right)>1\right\}} \\
& =\epsilon
\end{aligned}
$$


by the same reasoning used to establish (6.13) and (6.14). Combining (6.14) and (6.17) we see (ii) of Theorem 2.1 holds and hence $\left\{\mu_{k}\right\}$ is conditionally compact.

Remark 6.2. If $\left\{\mu_{k}\right\}$ is a sequence of probability measures on $E_{a}$ such that $\left\{\hat{\mu}_{k}\right\}$ converges uniformly on a set of the form $\left\{x \in E_{\alpha}^{\prime}:(T x, x) \leq 1\right\}$ where $T$ is an $S_{\alpha}$-operator then Theorem 6.2 implies $\left\{\mu_{k}\right\}$ is conditionally compact. Of course, if $\left\{\hat{\mu}_{k}\right\}$ converges uniformly on all of $E_{\alpha}^{\prime}$ it follows that $\left\{\mu_{k}\right\}$ is actually compact.

In our next result we obtain sufficient conditions for a complex valued function defined on $E_{\alpha}^{\prime}$ to be the Fourier transform of a probability on $E_{\alpha}$. In case $\alpha(t)=t^{2}$ then $E_{\alpha}=l_{2}$ and it is known [2] that they are necessary as well. If $a(t)=t^{p} \quad(1 \leq t<2)$ the sufficiency result is known from [1], but it is not known whether the conditions are necessary for $p<2$.

Theorem 6.3. Suppose (6.1), (6.2), (6.3), and (6.4) bold. Further, assume $\phi$ is a complex valued function dejined on $E_{\alpha}^{\prime}$ such that

(i) $\phi(0)=1, \phi$ is positive definite, and

(ii) $\phi$ is $\tau_{a}$-continuous.

Then there exists a probability measure $\mu$ on $E_{\alpha}$ sucb that $\hat{\mu}=\phi$.

Proof. Let $\left\{P_{n}\right\},\left\{Q_{n}\right\}$ denote the canonical projections on $E_{\alpha}$. Let $\mu_{k}$ denote the probability measure on $P_{k} E_{\alpha}$ whose Fourier transform is $\hat{\mu}_{k}(x)=$ $\phi\left({ }^{t} P_{k} x\right)$. Since $\left\{\hat{\mu}_{k}\right\}$ is then equicontinuous at zero in ${ }^{t} P_{m} E_{\alpha}^{\prime}$ for each integer $m$ it suffices to show (ii) of Theorem 2.1 holds. Take $\epsilon>0$ and choose an $S_{\alpha^{-}}$ operator such that $(T x, x) \leq 1$ implies $|1-\phi(x)|<\epsilon$. Now ${ }^{t} P_{m} y-{ }^{t} P_{n} y=$ ${ }^{t} P_{m}{ }^{t} Q_{n} y$ for $y \in E_{\alpha}^{\prime}$ and

$$
\begin{aligned}
& \underset{n}{\lim \sup _{k}} \lim _{m} \int_{E_{\alpha}^{\prime}}\left[1-\hat{\mu}_{k}\left({ }^{t} P_{m}{ }^{t} Q_{n} y\right)\right] P_{\lambda}(d y) \\
& =\lim _{n} \sup _{k} \lim _{m} \int_{E_{a}^{\prime}}\left[1-\phi\left({ }^{t} P_{k}{ }^{t} P_{m}{ }^{t} Q_{n} y\right)\right] P_{\lambda}(d y) \\
& \leq \epsilon+2 \lim _{n} \sup _{k} \lim _{m} P_{\lambda}\left\{x \in E_{a}^{\prime}:\left(T^{t} P_{k}{ }^{t} P_{m}{ }^{t} Q_{n} y,{ }^{t} P_{k}{ }^{t} P_{m}{ }^{t} Q_{n} y\right)>1\right\} .
\end{aligned}
$$

Choose $n_{0}$ by Lemma 6.1 such that for all $r, n \geq n_{0}$

$$
P_{\lambda}\left\{x \in E_{a}^{\prime}:\left(T^{t} P_{r}{ }^{t} Q_{n} x,{ }^{t} P_{r}{ }^{t} Q_{n} x\right)>1\right\}<\epsilon \text {. }
$$

Since ${ }^{t} P_{k}{ }^{t} P_{m}{ }^{t} Q_{n} y=0$ if $k \leq n$ and ${ }^{t} P_{r}{ }^{t} Q_{n} y$ where $r=\min (k, m)$ if $k>n$ we have by (6.18) and (6.19) that (ii) of Theorem 2.1 holds. Hence $\left(\mu_{k}\right)$ is conditionally compact and since $\lim _{k} \hat{\mu}_{k}=\phi$ on $E_{\alpha}^{\prime}$, i.e. ${ }^{t} P_{k} x \rightarrow x$ in the $\tau_{\alpha}$ topology for each $x$ in $E_{\alpha}^{\prime}$, it follows that $\left\{\mu_{k}\right\}$ converges weakly to a measure; call it $\mu$, and $\hat{\mu}=\phi$. Thus the theorem is proved. 
Remark 6.3. If the $S_{\alpha}$-operator $T$ chosen in the proof of Theorem 3.3 can be taken so that $t_{i j}=0$ for $i \neq j$ it is then possible to obtain Theorem $6.3 \mathrm{di}$ rectly from Theorem 6.1. That is, then $(T x, x) \leq 1$ implies $\left(T P_{k} x, P_{k} x\right) \leq 1$ for all $k=1,2, \ldots$ and hence $(6.11)$ holds for all $\hat{\mu}_{k}$ on $\{x:(T x, x) \leq 1\}$.

In case $E_{\alpha}=l_{2}$ it is known [2] that the conditions of Theorem 6.3 are necessary as well as sufficient.

\section{BIBLIOGR A PHY}

1. A. D. de Acosta, Existence and convergence of probability measures in Banach spaces, Trans. Amer. Math. Soc. 152 (1970), 273-298. MR $42 \# 2516$.

2. L. Gross, Harmonic analysis on Hilbert space, Mem. Amer. Math. Soc. No. 46 (1963), 62 pp. MR $28 \# 4304$.

3. M. A. Krasnosel'skií and Ja. B. Rutickiı, Convex functions and Orlicz spaces, Fizmatgiz, Moscow, 1959; English transl., Noordhoff, Groningen, 1961. MR 23 \#A4016.

4. J. Kuelbs, Positive definite symmetric functions on linear spaces, J. Math. Anal. Appl. (to appear).

5. J. Kuelbs and V. Mandrekar, Harmonic analys is on certain vector spaces, Trans. Amer. Math. Soc. 149 (1970), 213-231.

6. M. Loève, Probability theory, 3rd ed., Van Nostrand, Princeton, N. J., 1963. MR $34 \# 3596$.

7. L. LeCam, Remarques sur le théorème limite central dans les espaces localement convexes, Les Probabilités sur les Structure Algébriques, Colloq. Internat. du Centre National de la Recherche Scientifique, Paris, 1970, pp. 233-249.

8. S. Mazur and W. Orlicz, On some classes of linear spaces, Studia Math. 17 (1958), 97-119. MR 20 \#4780.

9. Ju. V. Prohorov, The method of characteristic functionals, Proc. Fourth Berkeley Sympos. Math. Statist. and Probability, vol. II, Univ. of California Press, Berkeley, Calif., 1961, pp. 403-419. MR 24 \#A3670.

10. A. C. Zaanen, Linear analysis. Measure and integral, Banach and Hilbert space, linear integral equations, Interscience, New York; North-Holland, Amsterdam; Noordhoff, Groningen, 1953. MR 15, 878.

11. H. W. Ellis and I. Halperin, Haar functions and the basis problem for Banach spaces, J. London Math. Soc. 31 (1956), 28-39. MR 17, 646.

DEPARTMENT OF MATHEMATICS, UNIVERSITY OF WISCONSIN, MADISON, WISCONSIN 53706 